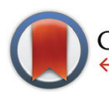

CrossMark \&lick for updates

Cite this: Dalton Trans., 2015, 44 966

Received 17th July 2014

Accepted 4th November 2014

DOI: $10.1039 / c 4 d t 02172 j$

www.rsc.org/dalton

\title{
A luminescence line-narrowing spectroscopic study of the uranium(vi) interaction with cementitious materials and titanium dioxide $\uparrow$
}

\author{
Jan Tits, ${ }^{{ }^{a}}$ Clemens Walther, $\$^{\mathrm{b}}$ Thorsten Stumpf, $\S^{\mathrm{b}}$ Nathalie Macé $\|^{\mathrm{a}}$ and \\ Erich Wieland ${ }^{\mathrm{a}}$
}

\begin{abstract}
Non-selective luminescence spectroscopy and luminescence line-narrowing spectroscopy were used to study the retention of $\mathrm{UO}_{2}{ }^{2+}$ on titanium dioxide $\left(\mathrm{TiO}_{2}\right)$, synthetic calcium silicate hydrate $(\mathrm{C}-\mathrm{S}-\mathrm{H})$ phases and hardened cement paste (HCP). Non-selective luminescence spectra showed strong inhomogeneous line broadening resulting from a strongly disordered $\mathrm{UO}_{2}{ }^{2+}$ bonding environment. This problem was largely overcome by using luminescence line-narrowing spectroscopy. This technique allowed unambiguous identification of three different types of $\mathrm{UO}_{2}{ }^{2+}$ sorbed species on $\mathrm{C}-\mathrm{S}-\mathrm{H}$ phases and HCP. Comparison with spectra of $\mathrm{UO}_{2}{ }^{2+}$ sorbed onto $\mathrm{TiO}_{2}$ further allowed these species to be assigned to a surface complex, an incorporated species and an uranate-like surface precipitate. This information provides the basis for mechanistic models describing the $\mathrm{UO}_{2}{ }^{2+}$ sorption onto $\mathrm{C}-\mathrm{S}-\mathrm{H}$ phases and $\mathrm{HCP}$ and the assessment of the mobility of this radionuclide in a deep geological repository for low and intermediate level radioactive waste (L/ILW) as this kind of waste is often solidified with cement prior to storage.
\end{abstract}

\section{Introduction}

Cementitious materials are commonly used worldwide for the solidification of low- and intermediate level radioactive waste (L/ILW) prior to storage in surface or deep geological repositories. ${ }^{1,2}$ For an accurate prediction of the long term fate of this radioactive waste, a comprehensive understanding of the chemical interactions of the radionuclides present in the waste with the solidification material is essential. In the past, radionuclide retention by cementitious materials has been typically attributed to adsorption on the surfaces of cement minerals (e.g. Evans ${ }^{3}$ and references therein). However, other immobilization processes, such as incorporation into the solid matrix, may take place. Incorporation processes give rise to the isolation of the radionuclide from the pore solution and may result in an irreversible immobilization in the solid matrix. The release of incorporated radionuclides is controlled by slow processes such as recrystallization and dissolution rather than

\footnotetext{
${ }^{a}$ Laboratory for Waste Management, Paul Scherrer Institute, CH-5232 Villigen PSI, Switzerland. E-mail: jan.tits@psi.ch

${ }^{b}$ Institute for Nuclear Waste Disposal, Karlsruhe Institute of Technology, D-76344 Eggenstein-Leopoldshafen, Germany

$\dagger$ Electronic supplementary information (ESI) available. See DOI: 10.1039/c4dt02172j $\$$ Present address: Institut für Radioökologie und Strahlenschutz, Leibnitz Universität Hannover, D-30419 Hannover, Germany.

$\S$ Present address: Helmholtz-Zentrum Dresden-Rossendorf, Institute of Resource Ecology, D-01314 Dresden, Germany.

ๆ Present address: CEA Saclay, F-91191 Gif-sur-Yvette CEDEX France.
}

by rapid surface desorption reactions. Calcium silicate hydrates (C-S-H) are major constituents of cementitious materials. They have a tobermorite-like layered structure consisting of $\mathrm{Ca}-\mathrm{O}$ sheets linked on each side to silicate chains in a "dreierkette" arrangement. ${ }^{4}$ These calcium silicate layers carry a net negative charge neutralised by either protons or $\mathrm{Ca}^{2+}$ cations in the interlayer. C-S-H phases are further characterized by high recrystallization rates making them an ideal system for radionuclide incorporation. Recently, several studies have found experimental evidence for the incorporation of trivalent and tetravalent actinides and lanthanides by substitution for $\mathrm{Ca}$ in the interlayers and the $\mathrm{Ca}-\mathrm{O}$ layer of C-S-H phases. ${ }^{5-9}$ It is still an open question whether or not larger actinyl ions such as $\mathrm{UO}_{2}{ }^{2+}$ exhibit similar incorporation behaviour.

Uranium is an important component of intermediate-level radioactive waste and the isotopes of this radionuclide contribute significantly to its long-term dose. ${ }^{10,11}$ Under the typical redox conditions prevailing in the alkaline environment of the cementitious near-field of an ILW repository $\left(E_{\mathrm{h}}=-0.23 \mathrm{~V}\right){ }^{12}$ uranium exists predominantly in the valence state $+\mathrm{VI}$ as the linear dioxo uranyl ion, $\mathrm{UO}_{2}{ }^{2+}$.

Sorption of hexavalent actinides $\left(\mathrm{UO}_{2}{ }^{2+}, \mathrm{NpO}_{2}{ }^{2+}\right)$ onto cementitious materials has been subject of several studies including macroscopic wet chemistry investigations as well as spectroscopic investigations: Batch sorption studies on C-S-H phases revealed fast sorption kinetics as steady state was reached within a period of approximately 5-10 days. ${ }^{13-15}$ 
Furthermore, sorption was found to be linear over a wide concentration range and highly dependent on the $\mathrm{pH}$ and the C-S-H composition. Indeed, measured solid-liquid distribution ratios $\left(R_{\mathrm{d}}\right)$ varied between $10^{6} \mathrm{~L} \mathrm{~kg}^{-1}$ at $\mathrm{pH}=10.0$ and $10^{2} \mathrm{~L}$ $\mathrm{kg}^{-1}$ at $\mathrm{pH}=13.3 .{ }^{14,15}$ Tits et al. ${ }^{14}$ further demonstrated that, in the case of $\mathrm{NpO}_{2}{ }^{2+}$, the observed dependence of $R_{\mathrm{d}}$ values on the C-S-H composition can almost entirely be explained by assuming competition between the sorption process and the formation of non-sorbing highly hydrolysed anionic $\mathrm{NpO}_{2}(\mathrm{OH})_{4}{ }^{2-}$ species in solution. ${ }^{14}$

Several attempts have been made to decipher the local coordination environment of $\mathrm{UO}_{2}{ }^{2+}$ sorbed onto cementitious materials with the help of X-ray absorption spectroscopy $(\mathrm{XAS})^{16-20}$ and luminescence spectroscopy. ${ }^{15}$ These studies revealed (1) the existence of at least two types of $\mathrm{UO}_{2}{ }^{2+}$ sorbed species, (2) a sorbed $\mathrm{UO}_{2}{ }^{2+}$ structural environment dominated by $\mathrm{O}, \mathrm{Si}$ and $\mathrm{Ca}$ atoms arranged in a similar way as in calcium uranyl silicates. The weak back-scattering contributions from neighbouring $\mathrm{Si}$ and $\mathrm{Ca}$ atoms of the $\mathrm{C}-\mathrm{S}-\mathrm{H}$ structure in the XAS spectra, however, prevented an unequivocal identification of the coordination environment and thus the determination of the dominant sorption process (surface complexation or incorporation, respectively). In addition, if more than one type of species is present, XAS data can only provide an averaged coordination environment. Hence, there is a need for complementary spectroscopic information about the coordination environment of sorbed $\mathrm{UO}_{2}{ }^{2+}$ in C-S-H phases with the aim of providing conclusive evidence for the significance of incorporation as an immobilization process for hexavalent actinides in cementitious materials.

Conventional non-selective luminescence spectroscopy is commonly applied to study the speciation of $\mathrm{UO}_{2}{ }^{2+}$ in both crystalline and amorphous solids (e.g., ref. 15, 21-28). However, this technique often gives rise to severe spectral inhomogeneous broadening effects, thus hiding valuable spectroscopic information from electronic and vibronic transitions. ${ }^{15,29,30}$ Inhomogeneous broadening occurs when $\mathrm{UO}_{2}{ }^{2+}$ ions occupy sites in amorphous host matrices or at the surface of solids. ${ }^{15,21,26,27}$ In both cases the local coordination environment of the luminescing species is ill-defined resulting in a significant variation of the energies of states of the sorbed $\mathrm{UO}_{2}{ }^{2+}$ ions. Although the electronic transition line for each single sorbed $\mathrm{UO}_{2}{ }^{2+}$ ion may be sharp, the position of the electronic transition line and the spacings between the vibronic lines will vary among non-equivalent sorbed $\mathrm{UO}_{2}{ }^{2+}$ ions, which results in inhomogeneously broadened overall electronic and vibronic transition bands composed of all the sharp lines of each single sorbed $\mathrm{UO}_{2}{ }^{2+}$ ion. Inhomogeneous line broadening can be reduced by applying site-selective excitation. ${ }^{29-34}$ This well-established technique is called luminescence line-narrowing spectroscopy (LLNS) and has been successfully applied for the detection and characterization of organic molecules and biomolecular systems (e.g. Jankowiak ${ }^{35}$ and references therein). A narrow-band tunable laser is used to selectively excite a small subset of a set of $\mathrm{UO}_{2}{ }^{2+}$ luminescence sites in a sample, at a frequency within the inhomogeneously broadened absorption band. The excited subset of $\mathrm{UO}_{2}{ }^{2+}$ ions decays radiatively to produce a well-resolved narrow-band spectrum allowing extraction of the spectroscopic information from the electronic and vibronic transitions. By scanning the laser source over the frequency range of the inhomogeneously broadened absorption band, all subsets of luminescing $\mathrm{UO}_{2}{ }^{2+}$ ions can be probed individually and the variation of the optical properties within the set of luminescing ions can be evaluated.

The aim of the present study is to determine the speciation of $\mathrm{UO}_{2}{ }^{2+}$ taken up by C-S-H phases in cementitious materials. To achieve this goal, the optical properties of $\mathrm{UO}_{2}{ }^{2+}$ sorbed on $\mathrm{C}-\mathrm{S}-\mathrm{H}$ phases and hardened cement paste (HCP) on the one hand side and of $\mathrm{UO}_{2}{ }^{2+}$ adsorbed onto the surface of titanium dioxide $\left(\mathrm{TiO}_{2}\right)$ on the other hand side, were investigated using LLNS. $\mathrm{UO}_{2}{ }^{2+}$ is known to sorb on $\mathrm{TiO}_{2}$ through the formation of inner-sphere surface complexes. ${ }^{25,36-38}$ Furthermore, $\mathrm{TiO}_{2}$ is a solid phase known to be stable under alkaline conditions and, in contrast to C-S-H phases, it is characterized by a low solubility and a low recrystallization rate. ${ }^{39}$ Therefore, $\mathrm{UO}_{2}{ }^{2+}$ incorporation into the structure of this mineral is unlikely, thus making it an ideal solid to study the optical properties of $\mathrm{UO}_{2}{ }^{2+}$ surface complexes under alkaline conditions. Differences in the luminescence spectra of $\mathrm{UO}_{2}{ }^{2+}$ sorbed on $\mathrm{TiO}_{2}$, $\mathrm{C}-\mathrm{S}-\mathrm{H}$ phases and HCP will enable us to determine whether or not this cation is incorporated in the C-S-H structure.

\section{Experimental}

\section{Materials}

All solutions and suspensions were prepared using reagentgrade chemicals and deionized, decarbonated water (Milli-Q water) generated by a Milli-Q Gradient A10 water purification system (Millipore Billerica, USA). Teflon containers and centrifuge tubes used for the preparation of the samples, were washed, left overnight in a solution of $0.1 \mathrm{M} \mathrm{HCl}$, and thoroughly rinsed with deionized water prior to use. Sample preparation was carried out in a glovebox under an inert nitrogen atmosphere $\left(\mathrm{CO}_{2}\right.$ and $\left.\mathrm{O}_{2}<2 \mathrm{ppm}\right)$.

\section{Analytical techniques}

Solution compositions were determined using an Applied Research Laboratory ARL 3410D inductively coupled plasma optical emission spectrometer (ICP-OES) or a Perkin Elmer ELAN 6100 inductively coupled plasma mass spectrometer (ICP-MS). A combination glass pH electrode (Metrohm, Switzerland) calibrated against dilute standard $\mathrm{pH}$ buffers $(\mathrm{pH}=7.0-11.0)$ was used to determine the $\mathrm{pH}$.

\section{Sample preparation}

An artificial cement pore water (ACW-I) was prepared based on an estimate of the $\mathrm{NaOH}$ and $\mathrm{KOH}$ concentration in a Portland cement porewater before any degradation of the material had occurred. ${ }^{40}$ It contains $0.18 \mathrm{M} \mathrm{KOH}$ and $0.114 \mathrm{M} \mathrm{NaOH}$ and has a $\mathrm{pH}$ of 13.3. A C-S-H phase with a $\mathrm{Ca}: \mathrm{Si}$ mol ratio $(\mathrm{C}: \mathrm{S})$ 
of 1.07 was synthesized in ACW-I using a method adapted from Atkins et al.: ${ }^{41}$ Silica fume (AEROSIL 300, Degussa-Huls AG, Baar Switzerland) was mixed with $\mathrm{CaO}$ in a polyethylene bottle at a weight ratio of $1: 1$. To this, ACW-I was added to achieve a solid to liquid $(\mathrm{S}: \mathrm{L})$ ratio of $2.5 \times 10^{-2} \mathrm{~kg} \mathrm{\textrm {L } ^ { - 1 }}$. A detailed characterization of this $\mathrm{C}-\mathrm{S}-\mathrm{H}$ phase is given in Tits et $a l .{ }^{42}$ After an ageing period of 4 weeks, the C-S-H suspension was ready for further use in sorption experiments.

A sulfate-resistant Portland cement (CEM I 52.5 N HTS) provided by Lafarge (France), was used to prepare the HCP samples. A modified ACW (ACW-II) ${ }^{40}$ with $\mathrm{pH}=13.3$ and simulating the composition of a pore water in equilibrium with fresh $\operatorname{HCP}\left([\mathrm{OH}]=0.3 \mathrm{M},[\mathrm{K}]_{\mathrm{t}}=0.18 \mathrm{M},[\mathrm{Na}]_{\mathrm{t}}=0.114 \mathrm{M},[\mathrm{Ca}]_{\mathrm{t}}=\right.$ $1.6 \times 10^{-3} \mathrm{M},[\mathrm{Al}]_{\mathrm{t}}=5 \times 10^{-5} \mathrm{M},[\mathrm{S}]_{\mathrm{t}}=2 \times 10^{-3} \mathrm{M},[\mathrm{Si}]_{\mathrm{t}}=5 \times$ $\left.10^{-5} \mathrm{M}\right)$ was added to the solid material to achieve the final $\mathrm{S}: \mathrm{L}$ of $2.5 \times 10^{-2} \mathrm{~kg} \mathrm{~L}^{-1}$.

$\mathrm{TiO}_{2}$ was obtained from Materion Advanced Chemicals Inc., Milwaukee, USA. This material is a mixture of $90 \%$ rutile and $10 \%$ anatase and has a specific surface area of $5 \pm 1 \mathrm{~m}^{2} \mathrm{~g}^{-1} \cdot{ }^{43} \mathrm{TiO}_{2}$ suspensions were prepared by mixing the appropriate amounts of $\mathrm{TiO}_{2}$ powder with ACW-I to obtain a $\mathrm{S}: \mathrm{L}$ ratio of $2.5 \times 10^{-2} \mathrm{~kg} \mathrm{L^{-1 }}$. The suspensions were equilibrated for 1 day, centrifuged at $4000 \mathrm{rpm}$ for 1 hour, and the supernatant solution replaced with fresh ACW-I. This washing procedure was repeated several times until the composition of the supernatant solution analysed by ICP-OES was constant and the $\mathrm{TiO}_{2}$ was in equilibrium with the ACW-I solution.

$0.1 \mathrm{~mL}$ of a $1.08 \times 10^{-2} \mathrm{M}$ uranyl nitrate solution in $10^{-3} \mathrm{M}$ $\mathrm{HNO}_{3}$ was added to $40 \mathrm{~mL}$ of the C-S-H, HCP and $\mathrm{TiO}_{2}$ suspensions. The $\mathrm{UO}_{2}{ }^{2+}$ doped suspensions were shaken end-overend for 30 days. After this equilibration period, phase separation of the solid and liquid phase was carried out by centrifugation at $4000 \mathrm{rpm}$ for 1 hour. The resulting wet pastes were transferred to a self constructed copper sample holder with a sapphire window, sealed with a Teflon disk and cooled to $<15 \mathrm{~K}$ by a helium refrigerated cryostat (CTI-cryogenics, USA) prior to luminescence measurements to reduce homogeneous spectral broadening. Determination of the $\mathrm{UO}_{2}{ }^{2+}$ concentration in the supernatant solution by ICP-MS showed that $>95 \%$ of the $\mathrm{UO}_{2}{ }^{2+}$ added to the C-S-H suspension, was retained on the solid phase resulting in a $\mathrm{UO}_{2}{ }^{2+}$ loading of $\sim 250 \mathrm{ppm}\left(\sim 10^{-3} \mathrm{~mol} \mathrm{~kg}^{-1}\right)$.

\section{$\mathrm{UO}_{2}{ }^{2+}$ luminescence spectroscopy}

$\mathrm{UO}_{2}{ }^{2+}$ luminescence is induced via charge-transfer excitation involving the transfer of an electron from the $\sigma_{\mathrm{u}}$ orbitals (mainly of the axial $\mathrm{O}^{2-}$ ) to $5 \mathrm{f}_{\delta}$ and $5 \mathrm{f}_{\phi}$ orbitals of the $\mathrm{U}^{6+}$ ion. ${ }^{44-46}$ The subsequent radiative relaxation gives rise to an electronic transition line, $\mathrm{E}$, having a frequency of radiation, $\nu_{\mathrm{E}}$, in the range $21000 \mathrm{~cm}^{-1}<\nu_{\mathrm{E}}<19000 \mathrm{~cm}^{-1}$. $\mathrm{UO}_{2}{ }^{2+}$ emission spectra are further characterized by a pronounced vibronic structure originating from the resolution of the vibrational energy levels in the ground state. In general, the totally symmetric stretch vibration $\left(\nu_{\mathrm{s}}\right)$ of the $\mathrm{UO}_{2}{ }^{2+}$ moiety is the most pronounced vibration mode and it appears as a series of homologous lines, $\mathrm{S}_{1-4}$, (vibronic progression) superimposed on $E$ (e.g. Fig. 2, spectrum a and $\mathrm{b}$ in the results section). The spacing $(\Delta)$ between $E$ and the first line of this series, $S_{1}$, results in values for $\nu_{\mathrm{s}}$ in the ground state as follows: $\nu_{\mathrm{s}}=$ $\sim 870 \mathrm{~cm}^{-1}$ for the $\mathrm{UO}_{2}{ }^{2+}$ aquo ion ${ }^{47,48}$ and $700 \mathrm{~cm}^{-1}<\nu_{\mathrm{s}}<$ $800 \mathrm{~cm}^{-1}$ for uranium minerals (silicates, carbonates) and $\mathrm{UO}_{2}{ }^{2+}$ sorbed species. ${ }^{26,28,49}$

$\mathrm{UO}_{2}{ }^{2+}$ luminescence spectra are usually measured after indirect $\mathrm{UO}_{2}{ }^{2+}$ excitation raising the molecule from the ground state to one of the upper electronic excited states. De-excitation follows in two steps: (1) radiationless de-excitation through internal conversion and vibrational relaxation to the lowest vibrational level of the first excited state and (2) relaxation from the first excited state to the ground state with the emission of light. In case of $\mathrm{UO}_{2}{ }^{2+}$ ions occupying a large range of sorption sites with slightly different bonding environments, this technique leads to the excitation of all $\mathrm{UO}_{2}{ }^{2+}$ luminescence sites within the inhomogeneously broadened absorption band resulting in poorly resolved broadened luminescence spectra. To reduce the effect of inhomogeneous broadening, LLN spectroscopy has been applied. The Jablonski diagram in Fig. 1 adapted from Jankowiak, ${ }^{35}$ illustrates the principle of this technique applied to $\mathrm{UO}_{2}{ }^{2+}$. In this diagram, the electronic ground state $(0,0)$ and the first electronic excited state $(1,0)$ of $\mathrm{UO}_{2}{ }^{2+}$ in an ill-defined coordination environment (e.g. an amorphous matrix) are shown. Each of these electronic states supports several vibrational energy levels (vibronic states) while, to simplify the diagram, only the first of them is shown for each electronic state $(0,1)$ and $(1,1)$. The variation of the energies of the excited state levels within the inhomogeneously broadened absorption band is represented by the slope, $\Gamma_{\mathrm{inh}}$ (Fig. 1).

Two different cases can be identified ${ }^{50}$ : (1) Resonant luminescence line-narrowing (red color in Fig. 1): A small subset of $\mathrm{UO}_{2}{ }^{2+}$ ions $\left(\mathrm{A}_{1}\right)$ is directly excited to the lowest vibrational level of the first excited state $(1,0)$ using an energy, $\nu_{\text {ex }}$, in the inhomogeneously broadened absorption band. These excited ions

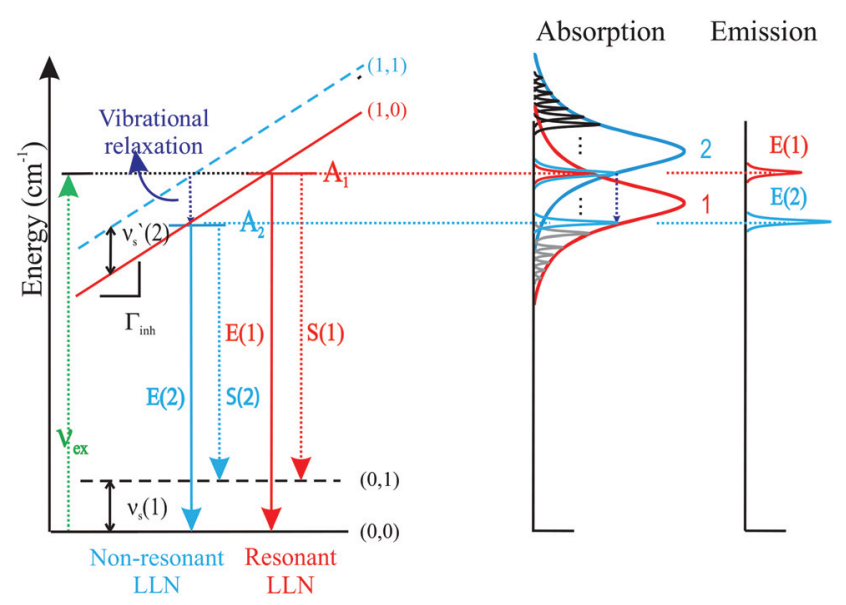

Fig. 1 Energy-level diagram illustrating resonant LLN E(1)) and nonresonant $\operatorname{LLN}(E(2))$ after site-selective laser excitation at an energy, $\nu_{\text {ex }}$ (diagram adapted from Jankowiak ${ }^{35}$ ). See text for a detailed explanation. 
decay radiatively directly to the ground state. The frequency, $\nu_{\mathrm{E}(1)}$, of the resulting narrow electronic transition line (E(1)), is identical to the frequency of the exciting laser light $\left(\nu_{\mathrm{ex}}=\nu_{\mathrm{E} 1}\right)$. (2) Non resonant line-narrowing (blue color in Fig. 1): Using the same excitation energy, $\nu_{\mathrm{ex}}$, another small subset of $\mathrm{UO}_{2}{ }^{2+}$ ions $\left(\mathrm{A}_{2}\right)$ may be excited to a higher-lying vibrational level of the first excited state $(1,1)$. From there, these ions decay nonradiatively to the lowest vibrational level of the first excited state by vibrational relaxation, followed by radiative decay to the ground state. The resulting narrow electronic line (E(2)) has a frequency, $\nu_{\mathrm{E}}(2)$, lower than $\nu_{\mathrm{E}}(1)$. It can be deduced from Fig. 1 that the spacing between $\mathrm{E}(1)$ and $\mathrm{E}(2)$ gives a value for the frequency of the totally symmetric stretch vibration of this second small subset of $\mathrm{UO}_{2}{ }^{2+}$ ions in the excited state, $\nu_{\mathrm{S}}^{\prime}(2)$. Non-resonant line-narrowing occurs when consecutive excited vibronic states of $\mathrm{UO}_{2}{ }^{2+}$ overlap due to inhomogeneous broadening.

In case $\mathrm{UO}_{2}{ }^{2+}$ clusters are formed in the sample, LLN spectra may be disturbed by homo-resonance energy transfer processes from the directly excited $\mathrm{UO}_{2}{ }^{2+}$ ions (donor ions) to nearby unexcited ions (acceptor ions) with slightly different transition frequencies (concentration quenching). ${ }^{51,52}$ This process is possible provided that the emission spectrum of the donor $\mathrm{UO}_{2}{ }^{2+}$ ion overlaps the absorption spectrum of the acceptor $\mathrm{UO}_{2}{ }^{2+}$ ion so that several vibronic transitions in the donor have similar energies as the corresponding transitions in the acceptor; i.e. the transitions are in resonance. This energy transfer results from long-range dipole-dipole interactions between donor and acceptor and not from the transfer of a photon. Following energy transfer, the acceptor ions may decay radiatively themselves or transfer their excitation energy to other nearby unexcited ions. The energy transfer efficiency depends on the extent of overlap between the donor emission spectrum and the acceptor absorption spectrum and on the donor-acceptor distance with an inverse $6^{\text {th }}$ power law. Homoresonance energy transfer occurs in samples with high concentrations of luminescence centers and is effective at donoracceptor distances up to $10 \mathrm{~nm}$ for systems with large absorption coefficients $(\varepsilon)$ and high luminescence quantum efficiencies $\left(\Phi_{0}\right) .{ }^{52}$ Absorption coefficients and quantum yields for $\mathrm{UO}_{2}{ }^{2+}$ luminescence strongly depend on the surrounding matrix, but they are generally quite low resulting in much smaller maximum distances for resonance energy transfer. Resonance energy transfer processes result in broadening of the transition lines. In extreme cases (e.g., the formation of $\mathrm{UO}_{2}{ }^{2+}$ precipitates), the resonant electronic transition line in LLN spectroscopy may completely disappear.

The $\mathrm{UO}_{2}{ }^{2+}$ luminescence lifetime depends partially on the presence of quenching ligands near the $\mathrm{UO}_{2}{ }^{2+}$ moiety. Shorter lifetimes are an indication for increased quenching. E.g. the luminescence of $\mathrm{UO}_{2}{ }^{2+}$ clusters exhibiting concentration quenching will typically be characterized by a shorter luminescence lifetime. Excited $\mathrm{UO}_{2}{ }^{2+}$ species typically decay monoexponentially and multi-exponential luminescence decay is often an indication for the presence of more than one luminescing species in a sample.
Luminescence spectroscopy was performed using a pulsed OPO laser system (Spectra Physics MOPO HF). Indirect excitation of $\mathrm{UO}_{2}{ }^{2+}$ was performed at an excitation energy of $24390 \mathrm{~cm}^{-1}$. The range used for the direct excitation was $21270 \mathrm{~cm}^{-1}<\nu_{\mathrm{ex}}<17857 \mathrm{~cm}^{-1}$. The luminescence signal was detected by an optical multichannel analyzer consisting of a Czerny Turner $300 \mathrm{~mm}$ polychromator (Jobin Yvon, HR 320 with gratings ranging from 300 to 1200 lines $\mathrm{mm}^{-1}$ ) and an intensified, gated photodiode array (Spectroscopy instruments, ST 180, IRY 700G) for the experiments with C-S-H phases and HCP, or a Czerny Turner spectrometer (Andor Shamrock with gratings ranging $150 / \mathrm{mm}-2400 / \mathrm{mm}$ ) coupled to an intensified ICCD (Andor Technologies) camera optimized for high on off ratio by use of a PROXITRONIC image intensifier for the experiments with $\mathrm{TiO}_{2}$. The laser light and Rayleigh and Raman scattering are filtered from the luminescence emission spectra by using a pulsed laser source and applying a minimum gate delay of $10 \mu$ s between the laser pulse and camera gating.

For the determination of the luminescence lifetime, luminescence decay profiles were recorded with a delay time step of $5 \mu \mathrm{s}$ or $25 \mu \mathrm{s}$ and a total of 90 steps were collected for each decay profile.

\section{Results and discussion}

\section{Non-selective luminescence spectroscopy of $\mathrm{UO}_{2}{ }^{2+}$ doped C-S-H phases}

Fig. 2c shows the normalized luminescence spectrum of uranyl ions sorbed onto C-S-H phases with a C:S ratio of 1.07 in ACW at a $\mathrm{pH}$ of 13.3 after indirect laser excitation at $\nu_{\mathrm{ex}}=$ $24390 \mathrm{~cm}^{-1}$. This spectrum is compared with the spectra of the free $\mathrm{UO}_{2}{ }^{2+}$ ion in $0.1 \mathrm{M} \mathrm{HNO}_{3}$ and an uranyl solution in 0.5 $\mathrm{M}$ tetramethyl ammonium hydroxide $(\mathrm{TMAOH})$ at $\mathrm{pH}=13.7$. Thermodynamic calculations performed using the NEA chemical

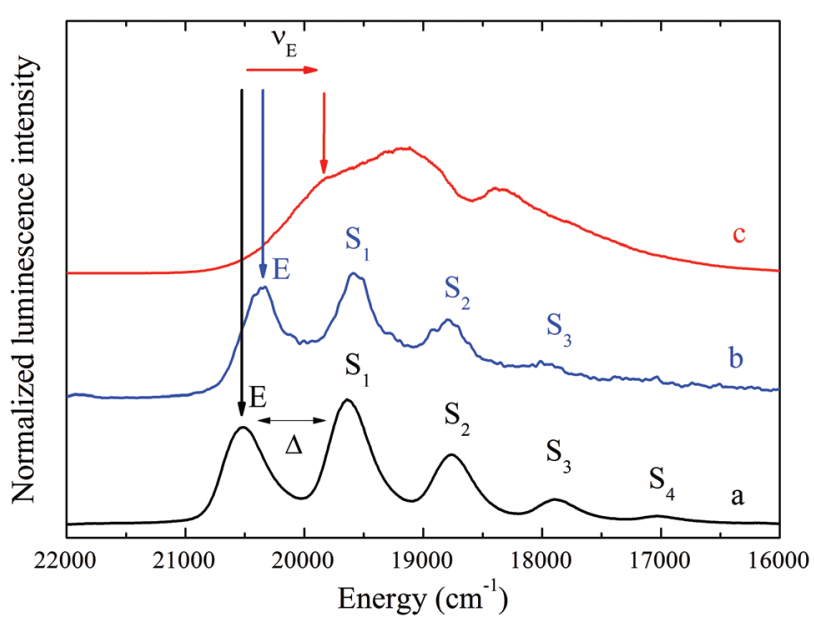

Fig. 2 Non-selective luminescence spectra recorded at $15 \mathrm{~K}$ of (a) $10^{-5}$ $\mathrm{MUO}_{2}{ }^{2+}$ in $0.1 \mathrm{M} \mathrm{HNO}_{3}, \mathrm{pH}=\sim 1.0$, (b) $10^{-5} \mathrm{M} \mathrm{UO}_{2}{ }^{2+}$ in $0.5 \mathrm{M} \mathrm{TMAOH}$, $\mathrm{pH}=13.7$, (c) $\mathrm{UO}_{2}{ }^{2+}$ sorbed onto $\mathrm{C}-\mathrm{S}-\mathrm{H}$ phases $(\mathrm{C}: \mathrm{S}=1.07$, in $\mathrm{ACW}$ at $\mathrm{pH}=13.3$ ); loading $=10^{-3} \mathrm{~mol} \mathrm{~kg}{ }^{-1} \cdot \nu_{\mathrm{ex}}=24390 \mathrm{~cm}^{-1}$. Delay time $=$ $10 \mu \mathrm{s}$, gate width $=1.0 \mathrm{~ms}$. 
thermodynamic database ${ }^{53}$ showed that the speciation of $\mathrm{U}(\mathrm{vI})$ in ACW is dominated by the strongly hydrolyzed $\mathrm{UO}_{2}(\mathrm{OH})_{4}{ }^{2-}$ complex. The uranyl-doped C-S-H phases exhibit weakly structured spectra similar to those reported in an earlier paper. ${ }^{15}$ The $\mathrm{UO}_{2}{ }^{2+}$ luminescence spectra are red shifted upon hydrolysis. This red-shift is even more pronounced for $\mathrm{UO}_{2}{ }^{2+}$ sorbed on C-S-H phases. It is caused by a significant weakening of the axial $\mathrm{U}=\mathrm{O}$ bonds upon sorption due to the increased electrondonating abilities of the ligands in the equatorial plane of the sorbed $\mathrm{UO}_{2}{ }^{2+}$ ions. ${ }^{15,48,54}$

The broad luminescence bands indicate strong variation in the bonding environment of the sorbed $\mathrm{UO}_{2}{ }^{2+}$ ions.

\section{LLN spectroscopy of $\mathrm{UO}_{2}{ }^{2+}$ doped C-S-H phases}

For the study of the sorption of $\mathrm{UO}_{2}{ }^{2+}$ onto C-S-H phases with LLN spectroscopy, three experimental parameters were varied: (1) The excitation energy $\left(\nu_{\mathrm{ex}}\right)$ : Variation of $\nu_{\mathrm{ex}}$ allows individual excitation of different subsets of $\mathrm{UO}_{2}{ }^{2+}$ luminescence centers within the inhomogeneously broadened electronic transition band, (2) the gate width (time period over which the luminescence signal is recorded) of the photodiode array: A change in the spectral shape with varying gate width is an indication for the presence of species with different lifetimes, (3) the delay time after the laser pulse: Luminescence signals recorded after increasing delay times after the laser pulse can be used for the construction of decay profiles allowing the determination of the lifetime of the excited state of a luminescing $\mathrm{UO}_{2}{ }^{2+}$ species.

Excitation spectra and LLN spectra of $\mathrm{UO}_{2}{ }^{2+}$ sorbed on C-S-H phases in ACW-I at $\mathrm{pH} 13.3$ were obtained by varying $\nu_{\mathrm{ex}}$ within the range of the inhomogeneously broadened electronic transition band $\left(\nu_{\mathrm{E}}\right)$ observed in the non-selective emission spectrum (21000 $\mathrm{cm}^{-1}-18000 \mathrm{~cm}^{-1}$; Fig. 2, spectrum c). At each excitation energy, a full emission spectrum was recorded and the luminescence intensity was integrated over the emission energy range between $22000 \mathrm{~cm}^{-1}$ and $16000 \mathrm{~cm}^{-1}$. The excitation spectra are poorly resolved due to the effect of inhomogeneous broadening and do not provide much spectral information. In the following, the excitation spectra of $\mathrm{UO}_{2}{ }^{2+}$ doped samples will therefore only be shown for completeness of the datasets, but they will not be considered for the spectral analysis.

Two series of luminescence spectra were recorded (Fig. 3). A gate delay time of $10 \mu \mathrm{s}$ was used in both series to filter out the emission interference originating from Rayleigh and Raman scattering. The gate width of the camera was fixed to $10 \mu \mathrm{s}$ in the first series and $10 \mathrm{~ms}$ in the second series. $\mathrm{UO}_{2}{ }^{2+}$ species with a shorter luminescence lifetime will dominate the spectra recorded with the small gate width whereas $\mathrm{UO}_{2}{ }^{2+}$ species with a longer lifetime will prevail in the spectra recorded with large gate width. The spectra of the first series are again characterized by broad, weakly resolved luminescence bands without any pronounced structure (Fig. 3a). The absence of a sharp luminescence line resonant with the energy of the laser excitation in these LLN spectra, is a strong indication for the presence of homo resonance energy transfer processes between adjacent sorbed $\mathrm{UO}_{2}{ }^{2+}$ moieties. The Förster distance (the distance between donor and acceptor at which the energy transfer efficiency is $50 \%$ ) for $\mathrm{UO}_{2}{ }^{2+}$ in $\mathrm{C}-\mathrm{S}-\mathrm{H}$ phases was estimated using the calculation procedure described by Hink et $a l .{ }^{55}$ and resulted in a value of $1.1 \mathrm{~nm}$ (see $\mathrm{ESI}_{\dagger} \dagger$ ). This observation suggests that at least one luminescent $\mathrm{UO}_{2}{ }^{2+}$ species in the $\mathrm{UO}_{2}{ }^{2+}$ doped C-S-H phases is present as a (surface) precipitate with neighbouring $\mathrm{UO}_{2}{ }^{2+}$ ions at distances closer than $\sim 1.1 \mathrm{~nm}$. Calculations using the NEA thermodynamic data for uranium $^{53}$ completed with solubility data for alkali uranates of Yamamura et al. ${ }^{56}$ and $\mathrm{CaUO}_{4}(\mathrm{~s})$ of Moroni et al. ${ }^{57}$ indicate that in ACW-I, $\mathrm{Na}_{2} \mathrm{U}_{2} \mathrm{O}_{7}$ should be the solubility controlling phase. The $\mathrm{UO}_{2}{ }^{2+}$ concentration in solution in equilibrium with the $\mathrm{UO}_{2}{ }^{2+}$ doped C-S-H phase was measured to be $7.0 \times 10^{-7} \mathrm{M}$, which is a factor of 10 below the solubility limit of $\mathrm{Na}_{2} \mathrm{U}_{2} \mathrm{O}_{7}$ in ACW $\left(6 \times 10^{-6} \mathrm{M}\right)$. Hence, $\mathrm{Na}_{2} \mathrm{U}_{2} \mathrm{O}_{7}$ precipitation in the $\mathrm{UO}_{2}{ }^{2+}$ doped C-S-H sample is not very probable and $\mathrm{UO}_{2}{ }^{2+}$ cluster formation must be catalyzed
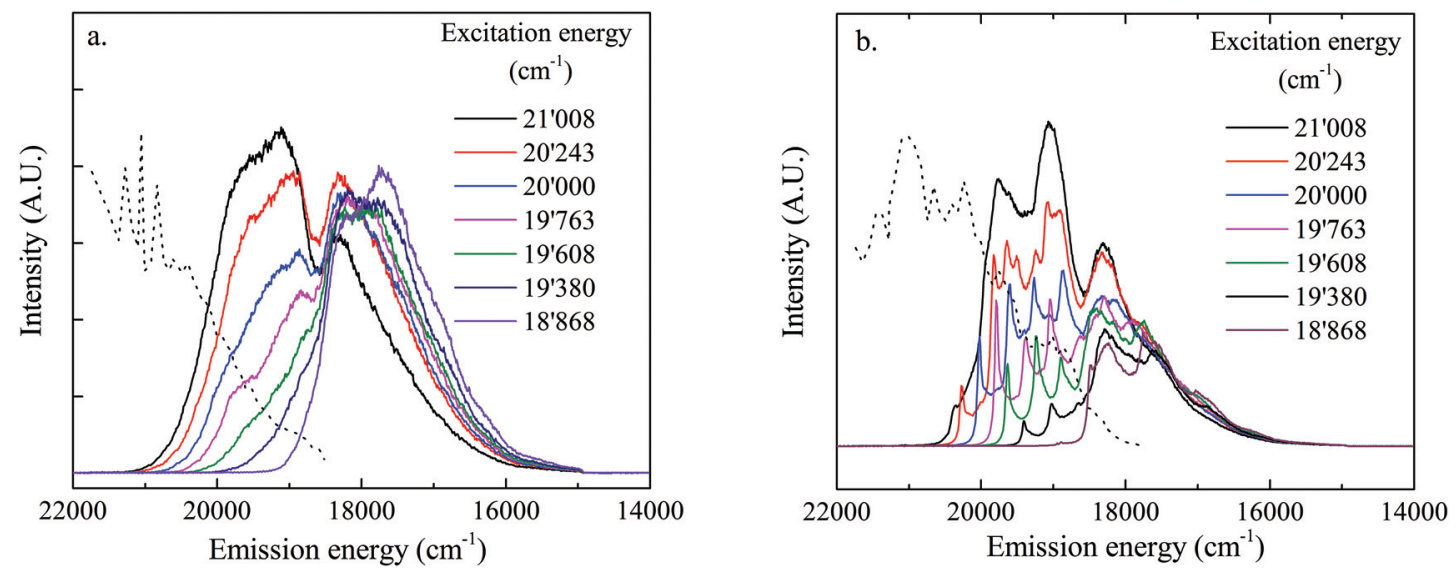

Fig. 3 Excitation spectra (dotted lines) and selected LLN spectra of $\mathrm{UO}_{2}{ }^{2+}$ sorbed on a $\mathrm{C}-\mathrm{S}-\mathrm{H}$ phase $(\mathrm{C}: \mathrm{S}=1.07)$ in $\mathrm{ACW}$ at $\mathrm{pH}=13.3$. UO ${ }_{2}^{2+}$ loading $=10^{-3} \mathrm{~mol} \mathrm{~kg}{ }^{-1}$. Excitation energy: $21008 \mathrm{~cm}^{-1}<\nu_{\mathrm{ex}}<18868 \mathrm{~cm}^{-1}$. Delay time $=10 \mu \mathrm{s}$. Gate width $=10 \mu \mathrm{s}(\mathrm{a})$ and $10 \mathrm{~ms}$ (b). The intensities of the excitation spectra in Fig. $3 \mathrm{a}$ and $3 \mathrm{~b}$ are divided by a factor 50 and 40 , respectively. 
by the C-S-H phase. On the other hand side, $\mathrm{U}-\mathrm{U}$ distances in uranates are in the range $3.5 \AA<\mathrm{R}_{\mathrm{U}-\mathrm{U}}<6.6 \AA \AA^{58}$ i.e., close enough for efficient homo resonance energy transfer.

The second series of luminescence spectra recorded with a larger gate width of $10 \mathrm{~ms}$ are characterized by a series of sharp luminescence bands in the frequency region $20500 \mathrm{~cm}^{-1}<$ $\nu<19000 \mathrm{~cm}^{-1}$. These spectra indicate the presence of at least one more $\mathrm{UO}_{2}{ }^{2+}$ sorbed species with a longer lifetime.

Furthermore, the spectra of this $\mathrm{UO}_{2}{ }^{2+}$ sorbed species appear to be less disturbed by energy transfer processes suggesting a more homogenous distribution of this $\mathrm{UO}_{2}{ }^{2+}$ sorbed species in the C-S-H sample. The first sharp line in each of these spectra is the luminescence line resonant with the laser excitation energy. It represents the electronic transition line of a subset of $\mathrm{UO}_{2}{ }^{2+}$ ions directly excited by the applied laser energy. The position of this line shifts with $\nu_{\text {ex }}$. The other sharp transition lines represent either non-resonant electronic transitions or vibronic transitions coupled to the electronic transitions.

A detailed spectral analysis was undertaken with the help of three typical emission spectra selected from the second series of spectra recorded with a large gate width (Fig. 4). The spectra were selected to include the spectrum measured with a high $\lambda_{\text {ex }}$, a spectrum measured with an intermediate $\nu_{\text {ex }}$ (arbitrarily chosen) and a spectrum measured with a low $\nu_{\text {ex }}$ having sufficient intensity to give an exploitable spectrum. The bands in each spectrum are assigned based upon their energy relative to the resonance line. The interpretation is based upon the Jablonski diagramme described in Fig. 1 and the assignment of the lines is summarized in Table 1.

The first line (1) is always the luminescence line resonant with the laser energy and represents the electronic transition (E(1)) of a small subset of $\mathrm{UO}_{2}{ }^{2+}$ sorbed species, U1. A series of homologous lines (vibronic progression) having a spacing, $\Delta$,

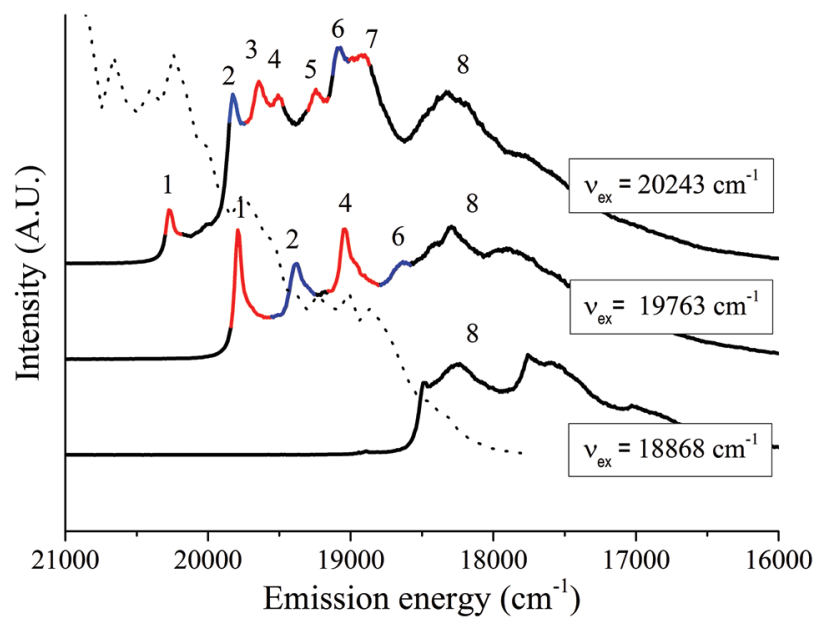

Fig. 4 Selected LLN spectra of $\mathrm{UO}_{2}{ }^{2+}$ sorbed on a C-S-H phase (C:S = 1.07) in $\mathrm{ACW}$ at $\mathrm{pH}=13.3$ recorded at three different excitation energies $\left(\nu_{\mathrm{ex}}=20243 \mathrm{~cm}^{-1}, 19763 \mathrm{~cm}^{-1}\right.$, and $\left.18868 \mathrm{~cm}^{-1}\right)$. Delay time $=10 \mu \mathrm{s}$. Gate width $=10 \mathrm{~ms}$. The numbered transition lines are described in the text and in Table 1. Red = lines assigned to species U1, blue = lines assigned to species U2.
Table 1 Assignment of spectral bands in Fig. 4

\begin{tabular}{ll} 
Number & Description \\
\hline$(1)$ & $\nu_{\mathrm{E}}(1):$ Resonant electronic transition line \\
$(2)$ & $\nu_{\mathrm{E}}(2):$ Non-resonant electronic transition line of species U1 \\
$(3)$ & $\nu_{\mathrm{E}}(3):$ Non-resonant electronic transition line of species U2 \\
$(4)$ & First line of vibronic progression on $\nu_{\mathrm{E}}(1)$ \\
$(5)$ & Could not be assigned \\
$(6)$ & First line of vibronic progression on $\nu_{\mathrm{E}}(2)$ \\
$(7)$ & First line of vibronic progression on $\nu_{\mathrm{E}}(3)$ \\
$(8)$ & Structureless spectrum of $\mathrm{UO}_{2}{ }^{2+}$ precipitate
\end{tabular}

between 700 and $800 \mathrm{~cm}^{-1}$, is associated with this electronic transition. These vibronic lines result from the coupling between the electronic transition and the totally symmetric stretch vibration in the ground state, $\nu_{\mathrm{s}}(1)$, of this subset. Indeed, the first line of this homologous series appears to be line (4) at a spacing $\Delta_{1-4}=758 \mathrm{~cm}^{-1}$ (Table 1). The other lines of this series are not resolved. Lines (2) and (3) in the spectra must represent non-resonant electronic transition lines $(\mathrm{E}(2)$ and $\mathrm{E}(3))$ of two more subsets of $\mathrm{UO}_{2}{ }^{2+}$ sorbed species. The first members of the vibronic progressions on each of these electronic transitions are the lines (6) and (7), with spacings $\Delta_{2-6}=744 \pm 10 \mathrm{~cm}^{-1}$ and $\Delta_{3-7}=758 \pm 10 \mathrm{~cm}^{-1}$, respectively. There are two possible interpretations for the presence of the two non-resonant electronic transitions lines (2) and (3): both subsets belong to the same set of $\mathrm{UO}_{2}{ }^{2+}$ sorbed species or both subsets belong to two entirely different sets of $\mathrm{UO}_{2}{ }^{2+}$ sorbed species.

(1) In the first case, the non-resonant lines (2) and (3) result from excitations to the second and third vibrational level of the first excited state $(1,1)$ and $(1,2)$, respectively, and the spacings $\Delta_{1-2}$ and $\Delta_{2-3}$, representing the frequencies of the totally symmetric stretch vibrations in the excited state $\left(\nu_{\mathrm{s}}^{\prime}(2)\right.$ and $\left(\nu_{\mathrm{s}}^{\prime}(3)\right)$, should be similar (see Fig. 1). The values of $\Delta_{1-2}$ and $\Delta_{2-3}$, however, were found to differ significantly; i.e., $406 \pm$ $10 \mathrm{~cm}^{-1}$ and $226 \pm 10 \mathrm{~cm}^{-1}$, respectively, indicating that this interpretation is wrong.

(2) Both subsets belong to two entirely different sets of $\mathrm{UO}_{2}{ }^{2+}$ sorbed species (U1 and U2) with clearly different coordination environments. In this case, the non-resonant lines (2) and (3) result from excitations to the second vibrational level of the first excited state of a subset of U1 and a subset of U2, respectively. The spectral characteristics of these two species are listed in Table 2. The main spectral difference between these two sets of $\mathrm{UO}_{2}{ }^{2+}$ sorbed species is the frequency of the symmetric stretch vibration in the excited state, $\nu_{\mathrm{s}}^{\prime}$, being $406 \pm$ $10 \mathrm{~cm}^{-1}\left(\Delta_{1-2}\right)$ and $632 \pm 10 \mathrm{~cm}^{-1}\left(\Delta_{1-3}\right)$ for U1 and U2, respectively.

The values of $\nu_{\mathrm{s}}$ and $\nu_{\mathrm{S}}^{\prime}$ found for the sorbed species U1 and $\mathrm{U} 2$ are significantly smaller than the values found for the $\mathrm{UO}_{2}{ }^{2+}$ aquo ion and the $\mathrm{UO}_{2}(\mathrm{OH})_{4}{ }^{2-}$ hydroxo species indicating a strong weakening of the axial $\mathrm{U}=\mathrm{O}$ bond $^{54}$ in agreement with the red-shift of the electronic transition line observed in the non-selective luminescence spectra in Fig. 2. This axial $\mathrm{U}=\mathrm{O}$ bond weakening results from increased $\sigma$ and $\pi$ donating 
Table 2 Spectral characteristics of two types of $\mathrm{UO}_{2}{ }^{2+}$ sorbed species obtained from LLN spectra recorded after excitation at $\nu_{\mathrm{ex}}=20243 \mathrm{~cm}^{-1}$, $19763 \mathrm{~cm}^{-1}$ and $18868 \mathrm{~cm}^{-1}$ (Fig. 4). Comparison with the spectral characteristics of the $\mathrm{UO}_{2}{ }^{2+}$ aquo ion and $\mathrm{UO}_{2}(\mathrm{OH})_{4}{ }^{2-}$ (literature data)

\begin{tabular}{lll}
\hline Aqueous uranyl species & $\nu_{\mathrm{s}} / \mathrm{cm}^{-1}$ & $\nu_{\mathrm{s}}^{\prime} / \mathrm{cm}^{-1}$ \\
\hline $\mathrm{UO}_{2}{ }^{2+}$ aquo ion & $870^{47,48}$ & $750^{44}$ \\
$\mathrm{UO}_{2}(\mathrm{OH})_{4}{ }^{2-}$ & $782^{47,48}$ & \\
\hline Type of $\mathrm{UO}_{2}{ }^{2+}$ sorbed species & $\nu_{\mathrm{S}} / \mathrm{cm}^{-1}$ & $\nu_{\mathrm{S}}^{\prime} / \mathrm{cm}^{-1}$ \\
\hline $\mathrm{U} 1$ & $\Delta_{1-4}=\Delta_{3-7}=758 \pm 10$ & $\Delta_{1-3}=632 \pm 10$ \\
$\mathrm{U} 2$ & $\Delta_{2-6}=744 \pm 10$ & $\Delta_{1-2}=406 \pm 10$ \\
$\mathrm{U} 3$ & - & -
\end{tabular}

abilities of the ligands coordinated to the $\mathrm{U}$ atom in the equatorial plane indicating strong bonding of the $\mathrm{UO}_{2}{ }^{2+}$ upon sorption. ${ }^{54}$

The LLN spectrum recorded at an excitation energy of $18868 \mathrm{~cm}^{-1}$ exhibits a completely different shape (Fig. 4). Similarly to the first series of LLN spectra recorded with short gate width (Fig. 3a), this spectrum is characterized by a few broad, weakly resolved luminescence bands without any pronounced structure. Furthermore, it does not contain a resonant electronic transition line. These spectral features are again a strong indication for the occurrence of energy transfer processes suggesting the presence of a (surface) precipitated $\mathrm{UO}_{2}{ }^{2+}$ species, U3. This observation confirms the assumption made for the interpretation of the first series of LLN spectra recorded with a short gate width. The strong red-shift of the spectrum of this surface precipitate is in agreement with luminescence spectra of alkaline-earth uranates described in the literature..$^{59,60}$ The poorly resolved transition bands of this spectrum don't allow the spectral characteristics of this species, $\nu_{\mathrm{s}}$ and $\nu_{\mathrm{s}}^{\prime}$ to be determined. The spectral characteristics of the three sorbed $\mathrm{UO}_{2}{ }^{2+}$ species, U1, U2 and $\mathrm{U} 3$, deduced from the LLN spectra, are summarized in Table 2.

In Fig. 5, the values of $\nu_{\mathrm{s}}$ and $\nu_{\mathrm{s}}^{\prime}$ for $\mathrm{U} 1$ and $\mathrm{U} 2$ obtained from the spacings $(\Delta)$ between the different transition lines in all the luminescence spectra recorded after excitation with increasing $\nu_{\mathrm{ex}}$, are plotted against $\nu_{\mathrm{ex}}$. This plot reveals that the frequencies of $\nu_{\mathrm{s}}$ and $\nu_{\mathrm{s}}^{\prime}$ for $\mathrm{U} 1$ and $\mathrm{U} 2$ decrease with increasing $\nu_{\text {ex }}$. This means that the $\mathrm{U}=\mathrm{O}$ axial distances of the subsets of $\mathrm{U} 1$ and $\mathrm{U} 2$, measured at higher excitation energies, are longer than the $\mathrm{U}=\mathrm{O}$ axial distances of the subsets measured at lower excitation energies. This observation is in agreement with the decreasing values of $\nu_{\mathrm{E}}$ for these subsets of $\mathrm{U} 1$ and $\mathrm{U} 2$ with increasing $\nu_{\mathrm{ex}}$ and is an expression of the variability of the bonding environments within the two types of species, U1 and $\mathrm{U} 2$.

In order to further check the existence of the postulated three different types of sorbed $\mathrm{UO}_{2}{ }^{2+}$ species $\mathrm{U} 1, \mathrm{U} 2$ and $\mathrm{U} 3$, the lifetimes of the different transition lines in the luminescence spectrum obtained after excitation at an energy of $19763 \mathrm{~cm}^{-1}$ were determined. Transition lines with the same lifetime are attributed to the same type of $\mathrm{UO}_{2}{ }^{2+}$ sorbed species, whereas transition lines with different lifetimes are

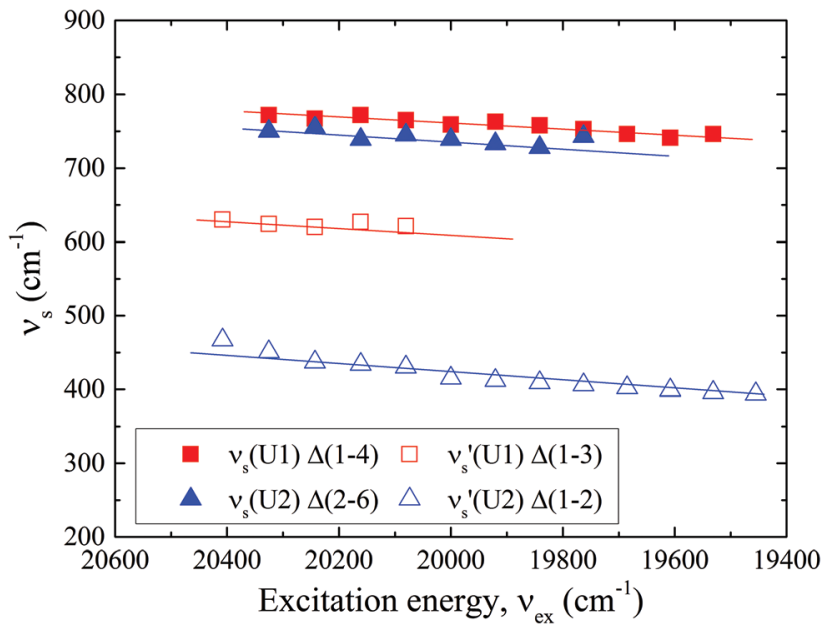

Fig. 5 Values for the $\mathrm{UO}_{2}{ }^{2+}$ totally symmetric stretch vibration in the ground state, $\nu_{\mathrm{s}}$, and in the excited state, $\nu_{\mathrm{s}}^{\prime}$, for subsets of the sorbed $\mathrm{UO}_{2}{ }^{2+}$ species $\mathrm{U} 1$ and $\mathrm{U} 2$, as function of the excitation energy, $\nu_{\mathrm{ex}}$.

attributed to another type of sorbed species. Decay profiles measured for the three sharp lines $(1,2$, and 4 in Fig. 4$)$ and the broad band (8 in Fig. 4) at higher energy are very similar; $i$. $e$., they can be fitted with a bi-exponential decay model suggesting the presence of at least two species with lifetimes of $108 \pm 31 \mu$ s and $275 \pm 31 \mu$ s, respectively (Fig. 6a, Table 3).

The short lifetime is attributed to the uranium (surface) precipitate, U3, as this species is also dominantly present in the series of spectra recorded with a short gate width (Fig. 3a). This lifetime corresponds very well with the lifetime of $100 \mu \mathrm{s}$ measured at $4.2 \mathrm{~K}$ by De Jong et al. ${ }^{60}$ for $\mathrm{MgUO}_{4}(\mathrm{~s})$, an uranate having a structure very similar to the structure of the Cauranate $\left(\mathrm{CaUO}_{4}(\mathrm{~s})\right)$ expected to precipitate at the surface of $\mathrm{C}-\mathrm{S}-\mathrm{H}$ phases in the present system.

Unfortunately, the difference in lifetimes of the two other species is too small to be detected with a decay profile analysis. However, a close inspection of the spectra recorded at different delay times, normalized to the resonant luminescence line, clearly shows that line (1) and line (4) decay at exactly the same rate, whereas line (2) exhibits a slower decay (the line rises relative to the first line with increasing delay time) (Fig. 6b). Furthermore, the broad band (8) at lower frequency exhibits a faster decay (the band decreases relative to the first line with increasing delay time). This observation clearly confirms that transition lines (1) and (4) belong to the same set of $\mathrm{UO}_{2}{ }^{2+}$ sorbed species (U1) whereas transition line (2) belongs to an additional set of species (U2) with a longer luminescence lifetime relative to that of U1. The longer lifetime indicates that non-radiative de-excitation processes for this set of species are less efficient due to the presence of a lower number of quenchers (e.g. $\mathrm{H}_{2} \mathrm{O}$ molecules) in its proximity. The broad transition band at higher frequencies belongs to the (surface) precipitate, U3, with a shorter luminescence lifetime relative to that of $\mathrm{U} 1$.

In summary, LLN spectroscopy of $\mathrm{UO}_{2}{ }^{2+}$ sorbed on a C-S-H phase in ACW at $\mathrm{pH}=13.3$ allowed three different sets of 

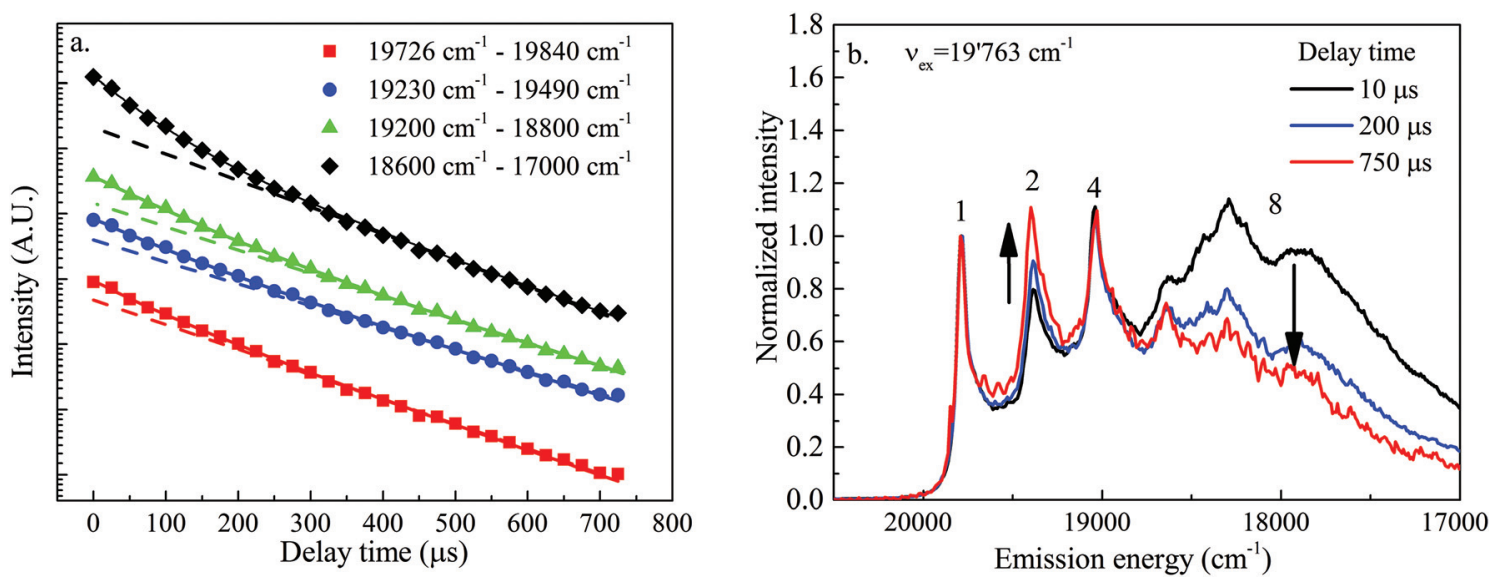

Fig. 6 (a) Luminescence lifetimes of different emission lines after direct excitation at $\nu_{\mathrm{ex}}=19763 \mathrm{~cm}^{-1}$. (b) LLN spectra recorded after excitation at $\nu_{\mathrm{ex}}=19763 \mathrm{~cm}^{-1}$ using three different delay times. Gate width $=10 \mathrm{~ms}$.

Table 3 Lifetimes of four transition bands determined from a decay profile analysis of LLN spectra obtained after excitation at an excitation energy of $\nu_{\mathrm{ex}}=19763 \mathrm{~cm}^{-1}$. Gate width $=10 \mathrm{~ms}$

\begin{tabular}{lll}
\hline Line number: & $t_{1}(\mu \mathrm{s})$ & $t_{2}(\mu \mathrm{s})$ \\
\hline $1\left(19726 \mathrm{~cm}^{-1}-19840 \mathrm{~cm}^{-1}\right)$ & 106 & 263 \\
$2\left(19230 \mathrm{~cm}^{-1}-19490 \mathrm{~cm}^{-1}\right)$ & 120 & 297 \\
$4\left(19200 \mathrm{~cm}^{-1}-18800 \mathrm{~cm}^{-1}\right)$ & 125 & 286 \\
$8\left(18600 \mathrm{~cm}^{-1}-17000 \mathrm{~cm}^{-1}\right)$ & 81 & 256 \\
Mean and 95\% confidence interval & $108 \pm 31$ & $275 \pm 31$ \\
using "Student's $t$ " numbers & &
\end{tabular}

species, U1, U2 and U3 to be identified. One of these species (U3) is a (surface) precipitate. This conclusion is in agreement with the results from previous time-resolved non-selective luminescence studies on a large number of C-S-H phases with varying compositions. ${ }^{15}$

Comparison with $\mathrm{UO}_{2}{ }^{2+}$ surface complexation on $\mathrm{TiO}_{2}$ and with $\mathrm{UO}_{2}{ }^{2+}$ sorbed on $\mathrm{HCP}$ : evidence for $\mathrm{UO}_{2}{ }^{2+}$ incorporation in $\mathrm{C}-\mathrm{S}-\mathrm{H}$ phases

LLN spectra of $\mathrm{UO}_{2}{ }^{2+}$ sorbed on $\mathrm{TiO}_{2}$ (loading $=10^{-3} \mathrm{~mol} \mathrm{~kg}{ }^{-1}$ ) in the presence of $\mathrm{ACW}$ at $\mathrm{pH}=13.3$ were recorded with a delay time of $10 \mu \mathrm{s}$ and using a gate width of $10 \mathrm{~ms}$ (Fig. 7a). Reducing the gate width to $10 \mu$ s did not have a significant effect on the shape of the LLN spectra. This is in contrast to the observations made with samples containing $\mathrm{UO}_{2}{ }^{2+}$ sorbed onto $\mathrm{C}-\mathrm{S}-\mathrm{H}$ phases for which a reduction of the gate width resulted in the disappearance of the resonance transition line and in a significant loss of spectral resolution caused by energy transfer between neighbouring $\mathrm{UO}_{2}{ }^{2+}$ ions due to the formation of an uranate-like (surface) precipitate. This observation suggests that such a precipitate with a short-lived luminescence signal did not form in the $\mathrm{TiO}_{2}$ sample although the aqueous chemistry and the total $\mathrm{UO}_{2}{ }^{2+}$ concentrations in the samples are identical with those in the C-S-H sample. Hence, it appears that an uranate-like (surface) precipitate is only formed on the surface of C-S-H phases. The LLN spectra of $\mathrm{UO}_{2}{ }^{2+}$ sorbed on $\mathrm{TiO}_{2}$ contain resonant electronic transition lines albeit much broader than in the case of $\mathrm{UO}_{2}{ }^{2+}$ sorbed onto C-S-H phases. Furthermore, the other transition bands observed in the LLN spectra are also much broader. Both observations suggest the presence of a significant homo resonance energy transfer from excited $\mathrm{UO}_{2}{ }^{2+}$ ions to neighbouring unexcited ions with slightly different bonding environments. Note that the presence of closely neighbouring $\mathrm{UO}_{2}{ }^{2+}$ atoms is not due to the formation of a $\mathrm{UO}_{2}{ }^{2+}$ precipitate as the spectral features of such a precipitate (i.e., the short-lived broad structureless peak at $18500 \mathrm{~cm}^{-1}>\nu_{\mathrm{E}}>17500 \mathrm{~cm}^{-1}$ ) is absent. A $\mathrm{UO}_{2}{ }^{2+}$ loading of $10^{-3} \mathrm{~mol} \mathrm{~kg}^{-1}$ on a $\mathrm{TiO}_{2}$ solid phase having a specific surface area of $\sim 5 \mathrm{~m}^{2} \mathrm{~g}^{-1}$ (ref. 43 ) means that approximately $12 \mathrm{UO}_{2}{ }^{2+}$ atoms are adsorbed on a surface area of $10 \mathrm{~nm}^{2}$. If the sorbed $\mathrm{UO}_{2}{ }^{2+}$ is homogeneously distributed over the $\mathrm{TiO}_{2}$ surface, the distance between adjacent sorbed $\mathrm{UO}_{2}{ }^{2+}$ ions is $\sim 3 \mathrm{~nm}$. A rough estimate of the Förster distance in this system gives a value of $\sim 0.7 \mathrm{~nm}$ (see ESI $\dagger$ ), significantly shorter than the distance of $3 \mathrm{~nm}$ calculated based upon the $\mathrm{UO}_{2}{ }^{2+}$ loading on the solid. Hence, homo resonance energy transfer cannot explain the line broadening observed in this sample. We don't have an explanation for this observation at present.

At low excitation energy $\left(18868 \mathrm{~cm}^{-1}\right)$ only the laser line is visible in the luminescence spectra. In contrast to the sample with $\mathrm{UO}_{2}{ }^{2+}$ sorbed on C-S-H phases, no luminescence signal is detected after excitation at this energy, confirming the absence of an uranate-like precipitate.

LLN spectra of $\mathrm{UO}_{2}{ }^{2+}$ sorbed on HCP again exhibit better resolved spectra (Fig. 7b). A clear electronic transition line can be identified in each spectrum, followed by several additional well-resolved narrow bands. At high excitation energy $\left(18868 \mathrm{~cm}^{-1}\right)$ a luminescence spectrum similar to that for the $\mathrm{UO}_{2}{ }^{2+}$ loaded C-S-H sample is observed indicating the presence of an uranate-like (surface) precipitate. For further interpretation of the bands in these two samples, LLN spectra of $\mathrm{UO}_{2}{ }^{2+}-\mathrm{C}-\mathrm{S}-\mathrm{H}, \mathrm{UO}_{2}{ }^{2+}-\mathrm{TiO}_{2}$ and $\mathrm{UO}_{2}{ }^{2+}-\mathrm{HCP}$ recorded after excitation at $506 \mathrm{~nm}$ are compared in Fig. 8. The numbers used for the transition lines are the same as given in Fig. 4. 

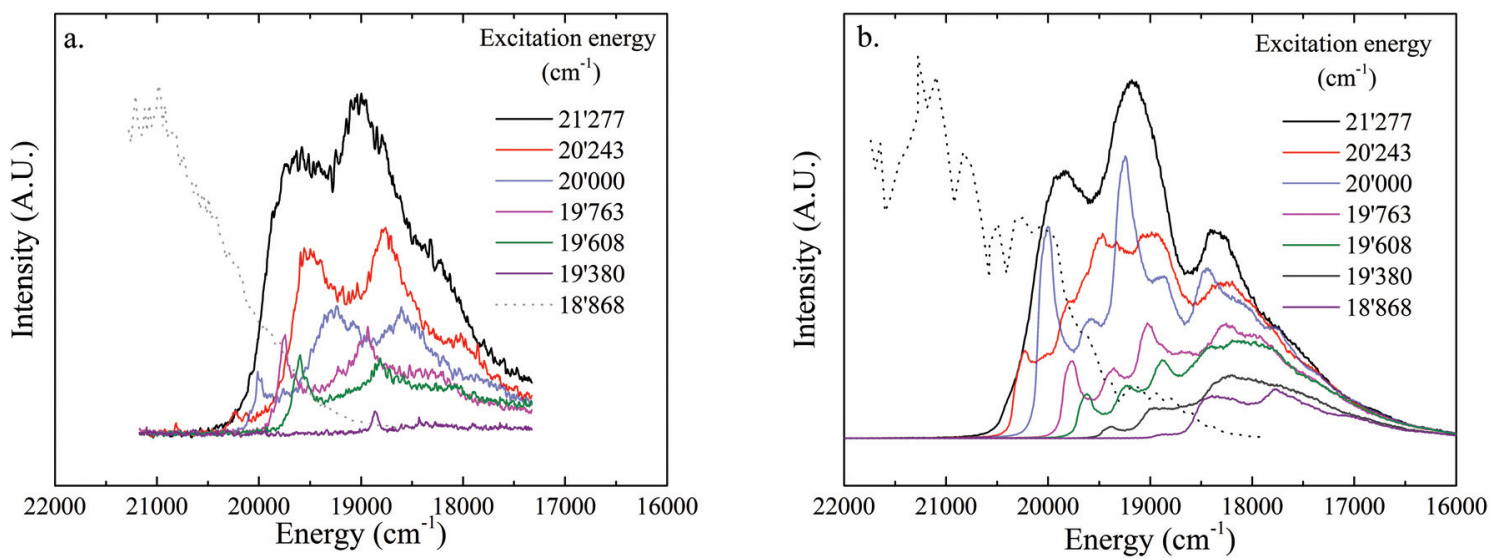

Fig. $7 \mathrm{LLN}$ spectra of $\mathrm{UO}_{2}{ }^{2+}$ sorbed on $\mathrm{TiO}_{2}$ (a) and on $\mathrm{HCP}(\mathrm{b})$ in the presence of ACW at $\mathrm{pH}=13.3$. Loading $=10^{-3} \mathrm{~mol} \mathrm{~kg}{ }^{-1}$. Excitation energy: $21008 \mathrm{~cm}^{-1}<\nu_{\mathrm{ex}}<18868 \mathrm{~cm}^{-1}$. Delay time $=10 \mu \mathrm{s}$. Gate width $=10 \mathrm{~ms}$. The intensities of the excitation spectra in Fig. $7 \mathrm{a}$ and $7 \mathrm{~b}$ are divided by a factor 500 and 200 , respectively.

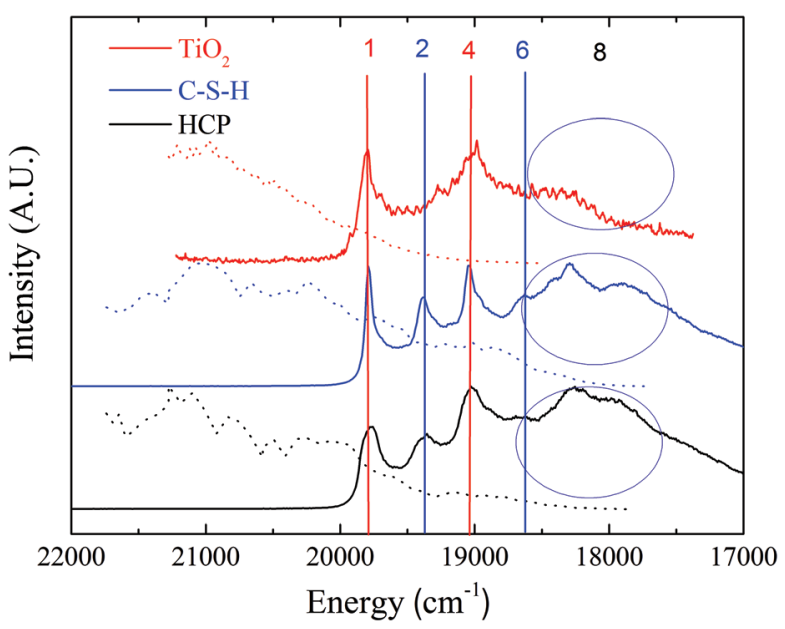

Fig. 8 LLN spectra of $\mathrm{UO}_{2}{ }^{2+}$ sorbed on $\mathrm{TiO}_{2}$, a $\mathrm{C}-\mathrm{S}-\mathrm{H}$ phase $(\mathrm{C}: \mathrm{S}=$ 1.07), and on $\mathrm{HCP}$ in $\mathrm{ACW}$ at $\mathrm{pH}=13.3 . \mathrm{UO}_{2}{ }^{2+}$ loading $=10^{-3} \mathrm{~mol} \mathrm{~kg}{ }^{-1}$. Excitation energy: $\nu_{\mathrm{ex}}=19763 \mathrm{~cm}^{-1}$. Delay time $=10 \mu \mathrm{s}$. Gate width $=$ $10 \mathrm{~ms}$. Numbered transition lines are described in the text.

Species U1 (red vertical lines) is found in the spectra of all three samples, although the spacing, $\Delta_{1-4}$, is slightly larger for the $\mathrm{U} 1$ species on $\mathrm{TiO}_{2}$ compared to the C-S-H sample and the HCP sample. We thus conclude that species U1 is a surface complex. Species U2 (blue vertical lines) is only present in the spectra of the C-S-H sample and the HCP sample and completely absent in the spectra of the $\mathrm{TiO}_{2}$ sample. The bands are sharp and well resolved indicating that energy transfer is not taking place so that this species must be homogeneously distributed in the C-S-H/HCP matrix. The absence of this sorbed species in the $\mathrm{TiO}_{2}$ sample, its homogeneous distribution in the C-S-H/HCP matrix and its longer lifetime suggesting a lower number of quenchers in close proximity, leads to the conclusion that these bands must belong to a $\mathrm{UO}_{2}{ }^{2+}$ species incorporated in the C-S-H structure in both samples.

The broad band typical for the uranate-like precipitate species, U3, in the frequency range, $18500 \mathrm{~cm}^{-1}<\nu<17000 \mathrm{~cm}^{-1}$ is only visible in the C-S-H and HCP samples and is completely absent in the $\mathrm{TiO}_{2}$ sample. This uranate-like solid phase must therefore be a Ca-uranate-like surface precipitate forming only on the Ca-rich C-S-H surfaces which are present in both the C-S-H and HCP samples.

\section{Conclusions}

LLN spectroscopy allows the unambiguous identification of three different types of sorbed $\mathrm{UO}_{2}{ }^{2+}$ species in C-S-H phases and in HCP.

Comparison of LLN spectra obtained for $\mathrm{UO}_{2}{ }^{2+}$ sorbed on C-S-H phases and HCP with LLN spectra obtained for $\mathrm{UO}_{2}{ }^{2+}$ surface complexes on $\mathrm{TiO}_{2}$ further allows the different types of sorbed species to be assigned to a surface complex, an incorporated species and a surface precipitate, respectively. Inhomogeneous broadening of absorption bands and luminescence bands after non-selective excitation indicates that all three sorbed $\mathrm{UO}_{2}{ }^{2+}$ species are characterized by a significant variation in their bonding environment.

LLN spectroscopy indicates that $\mathrm{UO}_{2}{ }^{2+}$ is retained in cement not only through adsorption on the surface of the solid cement components but also through incorporation into the structure of C-S-H phases. Similar incorporation processes were already observed in the past for trivalent and tetravalent actinides. Here, an unambiguous proof for the incorporation of the bulky $\mathrm{UO}_{2}{ }^{2+}$ cations in the C-S-H structure is presented. The study shows that $\mathrm{UO}_{2}{ }^{2+}$ sorbed on cement phases becomes isolated from the cement porewater. This suggests that the subsequent release from the cement phases is governed by dissolution or recrystallization processes of the C-S-H phases rather than by rapid surface desorption.

\section{Acknowledgements}

The authors would like to thank Dr M. Marques Fernandes (PSI) for the many helpful discussions. Thanks are extended to 
S. Büchner (Institute for Nuclear Waste Disposal, Karlsruhe Institute of Technology) for his invaluable assistance with the laser equipment. Partial financial support by the Swiss National Cooperative for the Disposal of Radioactive Waste (Nagra) is kindly acknowledged.

\section{Notes and references}

1 T. W. Hicks, T. D. Baldwin, P. J. Hooker, P. J. Richardson, N. A. Chapman, I. G. McKinley and F. B. Neall, Concepts for the geological disposal of intermediate-level radioactive waste, Galson Scienses Ltd report 0736-1, Version 1.1 on behalf of UK NDA, 2008.

2 F. P. Glasser, in Handbook of advanced radioactive waste conditioning technologies, ed. M. I. Ojovan, Woodhead, Oxford, UK, 2011, pp. 67-135.

3 N. D. M. Evans, Cem. Concr. Res., 2008, 38, 543-553.

4 I. G. Richardson, Cem. Concr. Res., 2008, 38, 137-158.

5 T. Stumpf, J. Tits, C. Walther, E. Wieland and T. Fanghänel, J. Colloid Interface Sci., 2004, 276, 118-124.

6 J. Tits, T. Stumpf, T. Rabung, E. Wieland and T. Fanghänel, Environ. Sci. Technol., 2003, 37, 3568-3573.

7 P. Mandaliev, T. Stumpf, J. Tits, R. Dähn, C. Walther and E. Wieland, Geochim. Cosmochim. Acta, 2011, 75, 2017-2029.

8 P. Mandaliev, E. Wieland, R. Dähn, J. Tits, S. V. Churakov and O. Zaharko, Appl. Geochem., 2010, 25, 763-777.

9 M. Schlegel, I. Pointeau, N. Coreau and P. Reiller, Environ. Sci. Technol., 2004, 38, 4423-4431.

10 D. McGinnes, Model radioactive waste inventory for reprocessing waste and spent fuel, Nagra Technical Report NTB 01-01, Nagra, Wettingen, Switzerland, 2002.

11 NAGRA, Project Opalinus Clay. Safety report. Demonstration of disposal feasibility for spent fuel, vitrified highlevel waste and long-lived intermediate level waste (Entsorgungsnachweis), Nagra Technical Report NTB 02-05, Nagra, Wettingen, Switzerland, 2002.

12 P. Wersin, L. H. Johnson, B. Schwyn, U. Berner and E. Curti, Redox conditions in the near-field of a repository for SF/HLW and ILW in Opalinus Clay, Nagra Technical Report NTB 02-13, Nagra, Wettingen, Switzerland, 2003.

13 I. Pointeau, C. Landesman, E. Giffault and P. Reiller, Radiochim. Acta, 2004, 92, 645-650.

14 J. Tits, X. Gaona, A. Laube and E. Wieland, Radiochim. Acta, 2014, 102, 385-400.

15 J. Tits, G. Geipel, N. Macé, M. Eilzer and E. Wieland, J. Colloid Interface Sci., 2011, 359, 248-256.

16 E. R. Sylwester, P. G. Allen, P. Zhao and B. E. Viani, Mater. Res. Soc. Symp. Proc., 2000, 608, 307-312.

17 P. Zhao, P. G. Allen, E. R. Sylwester and B. E. Viani, Radiochim. Acta, 2000, 88, 729-736.

18 M. Harfouche, E. Wieland, R. Dähn, T. Fujita, J. Tits, D. Kunz and M. Tsukamoto, J. Colloid Interface Sci., 2006, 303, 195-204.

19 N. Macé, E. Wieland, R. Dähn, J. Tits and A. Scheinost, Radiochim. Acta, 2013, 101, 379-389.
20 X. Gaona, E. Wieland, J. Tits, A. Scheinost and R. Dähn, Appl. Geochem., 2013, 28, 109-118.

21 C. J. Chisholm-Brause, J. M. Berg, K. M. Little, R. A. Matzner and D. E. Morris, J. Colloid Interface Sci., 2004, 277, 366-382.

22 H.-S. Chang, G. V. Korshin, Z. Wang and J. Zachara, Environ. Sci. Technol., 2006, 40, 1244-1249.

23 C. Götz, G. Geipel and G. Bernhard, J. Radioanal. Nucl. Chem., 2011, 287, 961-969.

24 G. Geipel, Coord. Chem. Rev., 2006, 250, 844-854.

25 J. Vandenborre, R. Drot and E. Simoni, Inorg. Chem., 2007, 46, 1291-1296.

26 Z. Wang, J. Zachara, J.-F. Boily, Y. Xia, T. C. Resch, D. A. Moore and C. Liu, Geochim. Cosmochim. Acta, 2011, 75, 2965-2979.

27 A. Kowal-Fouchard, R. Drot, E. Simoni and J. J. Ehrhardt, Environ. Sci. Technol., 2004, 38, 1399-1407.

28 Z. Wang, J. M. Zachara, P. L. Gassman, C. Liu, O. Qafoku, W. Yantasee and J. G. Catalano, Geochim. Cosmochim. Acta, 2005, 69, 1391-1403.

29 C. D. Flint, P. A. Tanner, R. Reisfeld and H. Tzehoval, Chem. Phys. Lett., 1983, 102, 249-253.

30 G. K. Liu, H. Z. Zhuang, J. V. Beitz, C. W. Williams and V. S. Vikhnin, Phys. Solid State, 2002, 44, 1433-1439.

31 M. Marques Fernandes, M. Schmidt, T. Stumpf, C. Walther, D. Bosbach, R. Klenze and T. Fanghänel, J. Colloid Interface Sci., 2008, 321, 323-331.

32 K. L. Lam and A. F. Leung, J. Non-Cryst. Solids, 1977, 23, 385-400.

33 A. F. Leung, J. Non-Cryst. Solids, 1976, 21, 41-46.

34 M. Marques Fernandes, T. Stumpf, B. Baeyens, C. Walther and M. H. Bradbury, Environ. Sci. Technol., 2010, 44, 921-927.

35 R. Jankowiak, in Shpol'skii spectroscopy and other site-selection methods, ed. C. Gooijer, F. Ariese and J. Hofstraat, Wiley-Interscience, New York, 2000, pp. 235-271.

36 C. Den Auwer, R. Drot, E. Simoni, S. D. Conradson, M. Gailhanou and J. Mustre de Leon, New J. Chem., 2003, 27, 648-655.

37 M. J. Comarmond, T. E. Payne, J. J. Harrison, S. Thiruvoth, H. Wong, R. D. Augterson, G. R. Lumpkin, K. Müller and H. Foerstendorf, Environ. Sci. Technol., 2011, 45, 55365542.

38 G. Lefèvre, J. Kneppers and M. Fédoroff, J. Colloid Interface Sci., 2008, 327, 15-20.

39 J. Schmidt and W. Vogelsberger, J. Solution Chem., 2009, 38, 1267-1282.

40 E. Wieland, J. Tits, A. Ulrich and M. H. Bradbury, Radiochim. Acta, 2006, 94, 29-36.

41 M. Atkins, F. P. Glasser and A. Kindness, Cem. Concr. Res., 1992, 22, 241-246.

42 J. Tits, E. Wieland, C. J. Müller, C. Landesman and M. H. Bradbury, J. Colloid Interface Sci., 2006, 300, 78-87.

43 K. Müller, H. Foerstendorf, T. Meusel, V. Brendler, G. Lefèvre, M. J. Comarmond and T. E. Payne, Geochim. Cosmochim. Acta, 2012, 76, 191-205.

44 C. Görller-Walrand, S. De Houwer, L. Fluyt and K. Binnemans, Phys. Chem. Chem. Phys., 2004, 6, 3292-3298. 
45 R. G. Denning, J. Phys. Chem. A, 2007, 111, 4125-4143.

46 S. P. McGlynn and J. K. Smith, J. Mol. Spectrosc., 1961, 6, 164-187.

47 C. Nguyen-Trung, D. A. Palmer, G. M. Begun, C. Pfeiffert and R. E. Mesmer, J. Solution Chem., 2000, 29, 101-129.

48 F. Quilès, C. Nguyen-Trung, C. Carteret and B. Humbert, Inorg. Chem., 2011, 50, 2811-2823.

49 Z. Wang, J. Zachara, J. P. McKinley and S. C. Smith, Environ. Sci. Technol., 2004, 39, 2651-2659.

50 G. F. Imbush, Phys. Scr., 1987, T19, 354-362.

51 J. R. Lakowicz, Principles of fluorescence spectroscopy, Springer Science+Business Media LLC, New-York, USA, 3rd edn, 2006.

52 B. Valeur, Molecular fluorescence: principles and applications, Wiley-VCH Verlag GmbH, Weinheim, 2001.

53 R. Guillaumont, T. Fanghänel, J. Fuger, I. Grenthe, V. Neck, D. A. Palmer and M. H. Rand, Chemical Thermodynamics
Vol. 5. Update on the Chemical Thermodynamics of Uranium, Neptunium, Plutonium, Americium and Technetium, Elsevier, North Holland, Amsterdam, The Netherlands, 2003.

54 S. Tsushima, Dalton Trans., 2011, 40, 6732-6737.

55 M. A. Hink, N. V. Visser, J. W. Borst, A. Van Hoek and A. J. W. Visser, J. Fluoresc., 2003, 13, 185-188.

56 T. Yamamura, A. Kitamura, A. Fukui, S. Nishikawa, T. Yamamoto and H. Moriyama, Radiochim. Acta, 1998, 83, 139-146.

57 L. P. Moroni and F. P. Glasser, Waste Manage., 1995, 15, 243-254.

58 D. M. Krol, P. M. Ros and A. Roos, J. Phys. Chem. A, 1980, 73, 1521-1526.

59 G. Blasse, Inorg. Chim. Acta, 1987, 129.

60 K. P. De Jong, D. M. Krol and G. Blasse, J. Lumin., 1978, 20, 241-248. 